\title{
Reliquidación de matrícula. Caracterización del beneficiario en la Universidad Francisco de Paula Santander
}

María Del Pilar Cárdenas Palomo, Mawency Vergel Ortega, Olga Lucy Rincón Leal

\section{Resumen}

Este trabajo analiza características de estudiantes de la Universidad Francisco de Paula Santander, conceptos de educación y equidad social. Una importante porción de la sociedad posee limitaciones para ingresar y/o permanecer en estudios de formación superior. Esta, como dificultad de ser "estudiante universitario", entendida como la "persona que cursa estudios en una institución de enseñanza superior", se destaca en los grupos de individuos con mayores desventajas económicas. Estos individuos se encuentran entrampados por habitar en un entorno desfavorable para estudiar y adquirir competencias. Esto perpetúa y acentúa las desigualdades sociales en el tiempo.

Palabras claves: Educación, socioeconómico, universidad

\section{Abstract}

This paper analyzes the characteristics of students from Francisco de Paula Santander, concepts of education and social equity University. A significant portion of society has limitations to enter and / or remain in advanced studies. This, as the difficulty of being "college student" understood as the "person who is studying at an institution of higher education", stands out in groups of individuals with greater economic disadvantages. These individuals are trapped by living in an unfavorable environment to study and acquire skills. This perpetuates and accentuates social inequalities over time.

Keywords: Education, socioeconomic, college

\section{Introducción}

$\mathrm{L}$ as políticas gubernamentales promueven el acceso a la educación superior y la equidad social. Por ello, las universidades semestre a semestre implementan acciones tendientes a la permanencia y graduación de los jóvenes que ingresan a sus programas académicos. No obstante, existen factores asociados que impiden que algunos estudiantes universitarios logren graduarse. Uno de los factores de deserción estudiantil hasta el 2012 en la Universidad Francisco de Paula Santander fue el factor socioeconómico; un $87 \%$ de los estudiantes pertenecen a un estrato 1 y 2 , y otros estudiantes universitarios inician sus estudios perteneciendo a un estrato socioeconómico

1 pililu_0417@hotmail.com, mawency@ufps.edu.co,olgarincon@ufps.edu.co

Universidad de Antioquia, Universidad Francisco de Paula Santander 


\section{Ecomatemático}

3, 4 o 6, pero diversas situaciones que inciden en su vida familiar, cambian el uso de sus recursos no permitiéndole continuar con sus estudios. De otra parte se encuentran registros en la Cámara de Comercio de años anteriores al ingreso a la Universidad que los sitúan en un estrato que realmente no les corresponde, lo cual con lleva al pago de matrículas elevadas que los padres no pueden sostener a lo largo de la vida académica del estudiante universitario.

Es por ello que la Universidad Francisco de Paula Santander, consiente del cumplimiento de su misión social, permite a los jóvenes solicitar reliquidación de matrícula como un medio para fomentar la permanencia, cuando considera debe ser revaluado el estrato en el cual ha sido ubicado y por consiguiente el valor de matrícula que viene cancelando. El presente estudio, caracteriza a los jóvenes de la Universidad Francisco de Paula Santander, su nivel socio económico y las percepciones que tienen del valor que cancelan.

\section{Contexto}

La Universidad Francisco de Paula Santander UFPS- con sede en Cúcuta, en el departamento de Norte de Santander, Colombia, es una institución de Educación Superior de carácter público al servicio de la región, sus dinámicas sociales, su situación fronteriza y del desarrollo de la comunidad; como lo evidencia su misión:

"La UFPS es una institución pública de educación superior, orientada al mejoramiento continuo y la calidad en los procesos de docencia investigación y extensión, cuyo propósito fundamental es la formación integral de profesionales, comprometidos con la solución de problemas del entorno, en busca del desarrollo sostenible de la región". ${ }^{1}$

La Vicerrectoría de Bienestar Universitario, según el Acuerdo 031 de 2011 adopta la política de bienestar y universidad saludable para contribuir a la formación integral, calidad de vida y conso- lidación de la comunidad universitaria, a través de programas y servicios permanentes, eficientes y eficaces, que posibilitan su desarrollo humano y el desarrollo cultural de la región. Una de las características que prima en esta instancia como dependencia administrativa es: la permanencia y graduación, que como política de las instituciones de educación superior, y compromiso de la Universidad Francisco de Paula Santander, pretende mejorar las condiciones de equidad social en el acceso y permanencia de los estudiantes, a través de programas y servicios diversificados, de amplia cobertura y calidad, que posibiliten disminuir las tasas de deserción, mejorar índices de desempeño académico y aumentar el índice de graduación de estudiantes.

"Concebir la Educación Superior como un derecho de la persona, un servicio público cultural, un proceso permanente que posibilita el desarrollo de las potencialidades del ser humano de una manera integral, con miras a configurar una sociedad más justa, equilibrada y autónoma, enmarcada dignamente en la comunidad internacional". ${ }^{2}$

Es así que la Vicerrectoría de Bienestar Universitario como ente garante de este tipo de servicios, crea el Programa de Caracterización socioeconómica de los estudiantes que solicitan reliquidación del valor de matrícula en la UFPS.

Las variables socioeconómicas que la institución considera para el estudio del valor de la matrícula estarán basados en:

"Las variables socioeconómicas que afectan la valoración del costo de la matricula son las siguientes: tipo de unidad económica familiar, lugar de residencia del estudiante, dependencia del estudiante con respecto a la familia de origen, carácter del colegio donde terminó el bachillerato, número de persona del grupo familiar que tienen condición de 


\section{Ecomatemático}

estudiantes, lugar de residencia de la familia de origen, nivel educativo del jefe de la familia de origen, posición socioeconómica del jefe de familia de origen, y patrimonio del grupo familiar". 3

Incrementar los índices de acceso a la educación superior en la ciudad, es un objetivo que como institución pública se promueve claramente, garantizando que las oportunidades y las condiciones de calidad de vida de la población aumenten, se garantice de esta forma su permanencia, y se aumente la exigencia académica, generando un trabajo continuo de cada uno de sus colaboradores, siendo de esta forma una institución con referentes importantes en la garantía del ejercicio académico y de la garantía del bienestar de su comunidad.

Bajo este marco normativo que emplea la UFPS como institución de educación superior, se crean espacios que garantizan desde diversos aspectos (económicos, sociales, políticos) el bienestar integral de su comunidad universitaria, que dependencias como la Vicerrectoría de Bienestar Universitario promueven desde cada uno de sus programas.

\section{Población}

La Universidad Francisco de Paula Santander cuenta con una población de 19053 estudiantes como institución de carácter público, de los cuales 14805 son estudiantes de la modalidad presencial en Cúcuta, población dentro de la cual se encuentran el 95\% de solicitudes de reliquidación de matrícula.

El proyecto tiene como beneficiarios fundamentalmente los y las estudiantes de los programas académicos en modalidad presencial, que estén cursando del II al X semestre del programa, y que soliciten verificar el valor de la matricula a la División de servicios Asistenciales y de Salud, dependencia adscrita a la Vicerrectoría de Bienestar Universitario de la Universidad Francisco de Paula Santander. En promedio cada semestre se reciben 150 solicitudes, a través de la vicerrectoría o a través de estímulo por pertenecer a grupos indígenas o afro descendientes. Para el presente estudio la población objeto de estudio la constituyeron 138 estudiantes.

\section{Justificación}

Los individuos de menores recursos tienen mayores dificultades para adquirir cualidades deseables para la empleabilidad. Mientras que su socialización primaria quizá no se desarrolle en un entorno propicio para la adquisición de dichas cualidades, su socialización secundaria (con una prácticamente única oportunidad en la universidad) se ve entorpecida por obstáculos económicos y sociales. Por ello, la búsqueda de la equidad en educación puede contribuir a que los jóvenes puedan revertir su situación de origen. Su búsqueda implica la necesidad de invocar dos principios de equidad horizontal "Igual trato para los iguales" y equidad vertical "desigual trato para los desiguales" (Morduchowicz 2003). Es decir, la educación y las instituciones educativas en el ámbito superior, deben prestar atención y tratar a cada individuo de acuerdo con su situación, lo cual implica la instauración de desigualdades.

La Vicerrectoría de Bienestar Universitario de la UFPS, y en línea con la normatividad nacional institucional, es una dependencia cuyo objetivo es de garantizar el bienestar integral a su comunidad universitaria, está en respuesta a las necesidades económicas que específicamente tienen los estudiantes de la institución para su acceso a la educación, que de la mano con la institución desde esta dependencia, y dando cumplimiento a la normativa de acceso a la educación en el País, se crean mecanismos como este proyecto de caracterización socioeconómica de los estudiantes que solicitan reliquidación del valor de la matrícula universitaria.

Desde esta perspectiva se permite generar alternativas de acceso a la educación superior, a través del reconocimiento de la historia de vida de los estudiantes de la institución, por parte de sus funcionarios quienes velarán porque este sea un proceso transparente, equitativo y correspondiente con la realidad de los beneficiarios; por tal razón 


\section{Ecomatemático}

la Vicerrectoría de Bienestar Universitario posibilita este proceso, pretendiendo consolidar aspectos importantes para el desarrollo de estrategias de acceso de la educación superior y mejoramiento de las condiciones de vida de la comunidad universitaria.

\section{Objetivo General}

Identificar las características económicas y sociales de los estudiantes universitarios a partir del II semestre de los programas académico modalidad presencial de la UFPS, que solicitan evaluar el costo asignado como matrícula académica.

\section{Método}

El estudio sigue un enfoque cuantitativo, método descriptivo, de tipo campo; se apoya en enfoque cualitativo para construcción a partir de hallazgos donde interactúa con los sujetos (estudiantes) y las realidades, por lo que fue necesario la realización de un proceso de planeación previo de la situación problema (lecturas previas, entrevistas y visitas institucionales), la determinación de la herramienta necesaria para el logro del objetivo del proyecto (capacitación y actualización de herramientas de intervención), y la ejecución de las estrategias definidas como trabajo de campo donde se aplica la herramienta principal del proyecto, la visita domiciliaria. Se diseñan instrumentos de entrevista y formato encuesta, con una fiabilidad alta de 0,87 y 0,81. El desarrollo de la propuesta metodológica y sus herramientas necesarias incluye para la universidad, capacitar periódicamente el personal a cargo del proyecto de reliquidación en busca de cualificar y unificar conceptos, y socializar el material actualizado que se emplea en el proyecto (manuales ${ }^{4}$, formatos ${ }^{5} y$ programas ${ }^{6},{ }^{7}$ con el fin de que exista un conoci- miento unificado y aplicación correcta del mismo, así como brindar el concepto profesional sobre los casos de los estudiantes a partir de un informe específico los hallazgos de la visita domiciliaria en relación a la condición socioeconómica de los estudiantes solicitantes, y realizar una reunión final con el personal vinculado al proceso para construir las conclusiones finales del proyecto.

Para la obtención de la información necesaria para este análisis, se toma como fuente secundaria el formato de reliquidación y los archivos adjuntos que los estudiantes anexaron al momento de hacer la solicitud, además de información institucional obtenida de la División de Sistemas. Y como fuente primaria, se obtiene información a través de las visitas domiciliarias

\section{Variables consideradas}

El ministerio de Tecnologías de la Información y las Comunicaciones, estipula una orientación que se retoma para este estudio referente a las variables que se deben considerar para la caracterización de usuarios o individuos beneficiarios de un servicio. El siguiente esquema evidencia los niveles de conocimiento que se requiere para el reconocimiento individual de los sujetos.

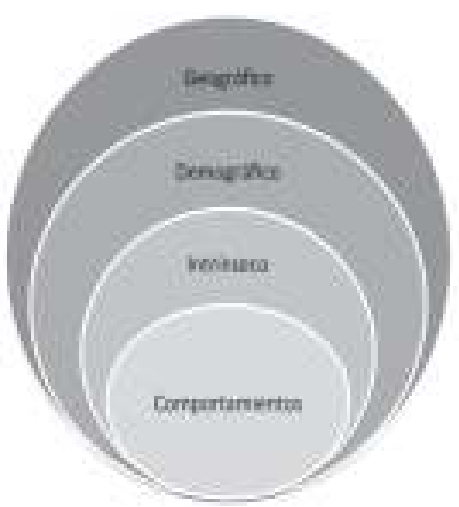

Gráfica 1. Niveles de conocimiento de ciudadanos particulares $^{8}$

${ }^{4}$ Anexo 1. Manual de funciones para la correcta implementación y desarrollo de visitas domiciliarias del proyecto de reliquidación del valor de matrícula académica. Trabajadora Social María del Pilar Cárdenas Palomo. 2013.

${ }^{5}$ Anexo 2. Formato de visita domiciliaria. Trabajadora Social María del Pilar Cárdenas Palomo. 2013

${ }^{6}$ Anexo 3. Proyecto "Caracterización socioeconómica del estudiante que solicita reliquidación del valor de matrícula de la Universidad Francisco de Paula Santander. Trabajadora Social María del Pilar Cárdenas Palomo. 2013.

${ }^{7}$ Anexo 4.Presentación de la capacitación "Herramientas teórico-prácticas para la implementación de visitas domiciliarias". Trabajadora Social María del Pilar Cárdenas Palomo. 2013.

${ }^{8}$ Ministerio de las Tecnologías de la información y las comunicaciones. República de Colombia. Gobierno en línea. Guía para la caracterización de usuarios de las entidades públicas. Tomado de: www.mintic.gov.co. Consultado el: 15 de Marzo de 2013. 


\section{Ecomatemático}

De acuerdo a esto y teniendo en cuenta orientaciones institucionales sobre la intención de reconocimiento de los estudiantes (variables socioeconómicas para el estudio del valor de la matrícula) ${ }^{9}$, se definieron variables de tres de los niveles de conocimiento que se desean profundizar de los estudiantes (geográficos, demográficos, e intrínsecos). La siguiente tabla se presenta las variables correspondientes a cada uno de los niveles que serán analizadas posteriormente:

\begin{tabular}{|c|c|c|}
\hline $\begin{array}{l}\text { GEOGRÁFI- } \\
\text { COS }\end{array}$ & $\begin{array}{l}\text { DEMOGRÁ- } \\
\text { FICOS }\end{array}$ & $\begin{array}{l}\text { INTRÍNSE- } \\
\text { COS }\end{array}$ \\
\hline $\begin{array}{l}\text { Lugar de resi- } \\
\text { dencia }\end{array}$ & Edad & $\begin{array}{l}\text { Programa acadé- } \\
\text { mico }\end{array}$ \\
\hline $\begin{array}{l}\text { Estrato socioeco- } \\
\text { nómico }\end{array}$ & Género & $\begin{array}{l}\text { Semestre acadé- } \\
\text { mico }\end{array}$ \\
\hline $\begin{array}{l}\text { Lugar de naci- } \\
\text { miento }\end{array}$ & Estado civil & $\begin{array}{l}\text { Carácter del } \\
\text { colegio del que } \\
\text { egresa }\end{array}$ \\
\hline Tipo de vivienda & $\begin{array}{l}\text { Tipología } \\
\text { familiar }\end{array}$ & $\begin{array}{l}\text { Porcentaje de } \\
\text { matrícula }\end{array}$ \\
\hline $\begin{array}{l}\text { Tenencia de la } \\
\text { vivienda }\end{array}$ & $\begin{array}{l}\text { Relaciones } \\
\text { familiares }\end{array}$ & \\
\hline $\begin{array}{l}\text { Servicios públi- } \\
\cos \end{array}$ & $\begin{array}{l}\text { Ocupación } \\
\text { laboral del } \\
\text { estudiante }\end{array}$ & \\
\hline $\begin{array}{l}\text { Materiales de la } \\
\text { vivienda }\end{array}$ & $\begin{array}{l}\text { Ingresos fami- } \\
\text { liares }\end{array}$ & \\
\hline
\end{tabular}

Tabla 1. Variables de análisis de la caracterización

\section{Resultados}

Percepciones en Educación y equidad. El nivel educativo se plantea como uno de los más importantes determinantes de su éxito en la vida futura. Pero consideran que no todos los individuos reciben la misma educación, los individuos más pobres poseen mayores dificultades para acceder a un nivel educativo alto; y puede concluirse que serán menos favorecidos en empleos lo cual genera poco éxito y baja calidad de vida. Y no sólo habrá individuos menos felices por baja cobertura en educación y empleo sino que dichos individuos serán los que provengan de hogares menos aventajados, por lo que puede pensarse que quedarán vinculados en un círculo de pobreza.

Una manera de cancelar la matrícula es la búsqueda de empleo en lo que pueda salir de primera mano, modelaje, ventas, organización de campeonatos, elaboración de trabajos a compañeros de clase, y en algunos casos compañía a otras parejas son algunos de los medios señalados por los estudiantes universitarios para conseguir dinero.

Por otra parte, quienes tienen bajos ingresos o viven en situaciones precarias (vivienda en invasión, o tablas, piso de tierra) continúan estudiando manifiestan en un $60 \%$ poseen un muy bajo rendimiento académico, al no estar bien alimentados no contar habitualmente con medios tecnológicos para elaborar trabajos, dialogar por chat para solicitar ayuda a compañeros y no poder concentrar su tiempo libre en el estudio, porque deben cuidar de hermanos menores, familiares mayores o realizar algún tipo de trabajo informal para subsistir. En muchos casos, la asistencia a la universidad se reduce a la búsqueda del servicio de restaurante, que puede llegar a representar el único alimento del día, o un jugo en la esquina, pan o una gaseosa.

La pobreza o el hecho de que familiares les retiren el apoyo en el estudio, consideran les genera rechazo social, se sienten frustrados, algunos manifiestan estar tentados a unirse a colectivos que se reúnen en el "aeropuerto". Un bajo porcentaje culpa a la universidad del valor a cancelar, como institución que les impide formarse. Se considera un factor a estudiar en detalle para prevenir problemas de integración social.

${ }^{9}$ Acuerdo 071 de 1990 de la UFPS. Artículo 15. Encontrado en: www.ufps.edu.co. Consultado el 20 de Enero de 2013: "Las variables socioeconómicas que afectan la valoración del costo de la matricula son las siguientes: tipo de unidad económica familiar, lugar de residencia del estudiante, dependencia del estudiante con respecto a la familia de origen, carácter del colegio donde terminó el bachillerato, número de persona del grupo familiar que tienen condición
de estudiantes, lugar de residencia de la familia de origen, nivel educativo del jefe de la familia de origen, posición socioeconómica del jefe de familia de origen, y patrimonio del grupo familiar". 


\section{Ecomatemático}

De esta manera, puede observarse cómo la educación superior se encuentra ligada a la estructura de oportunidades de la sociedad. Los más desfavorecidos, pertenecientes a los sectores de más bajos recursos, tienen menores oportunidades de adquirir competencias y alcanzar su formación profesional.

Variables intrínsecas: este tipo de variables posibilita a la dependencia institucional, reconocer el número de estudiantes por programa, semestre y porcentaje de matrícula que cancela actualmente el estudiante.

Programa académico por facultades al que pertenecen los estudiantes: del total (138) de estudiantes visitados en su domicilio para el proceso de reliquidación, el $42.06 \%$ pertenecen a programas de la Facultad de Ingeniería, principalmente de programas como Ingeniería Civil, Industrial y de Minas, seguido con un 39.85\% programas de la Facultad de Ciencias Empresariales, principalmente de programas como Administración de Empresas y Comercio Internacional.

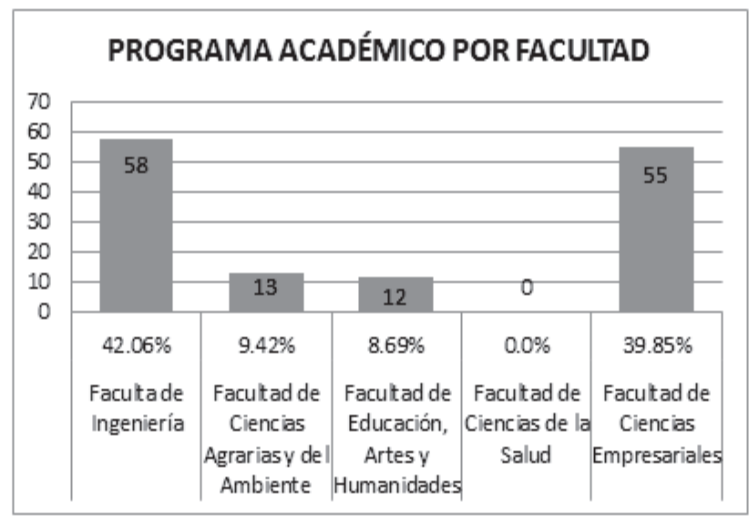

Gráfica 2. Programa académico por facultad

Semestre académico: en el nivel académico que se encuentran los estudiantes encontramos como los niveles más altos de participación, estudiantes cursantes del tercer semestre académico con una incidencia del $15.21 \%$, y del cuarto semestre académico con un $13.76 \%$, además de la falta de definición de este aspecto (sin dato) con el porcentaje más alto con un $36.23 \%$ del total de los estudiantes. Sin embargo con los índices más bajos encontramos estudiantes del décimo semestre con un $0.72 \%$ y del séptimo semestre con un $1.44 \%$.
Carácter del colegio del que egresa: el carácter del colegio del que egresan los estudiantes, es determinante del rango socioeconómico que se establece para el valor del pago de su matrícula, lo que indica que evidentemente el interés de estudiantes provenientes de educación pública para realizar la solicitud de reliquidación que con un $63.76 \%$ supera el $21.01 \%$ de educación privada.

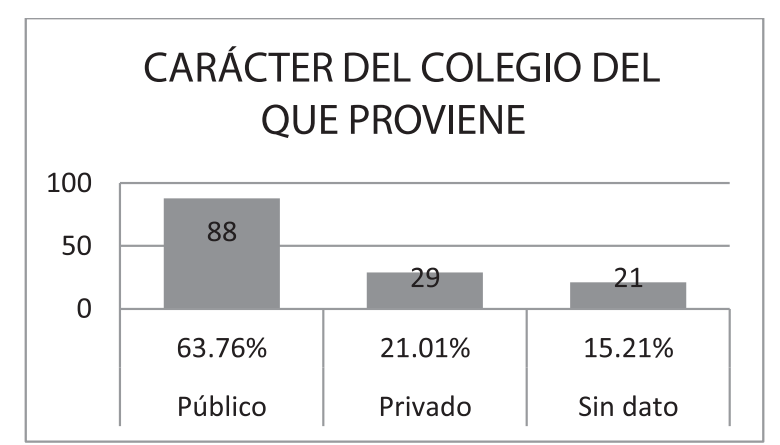

Gráfica 3. Identificación del colegio de procedencia

Porcentaje de matrícula que cancela: el porcentaje de matrícula se establece según las condiciones socioeconómicas del estudiante, y se estima según el valor del salario mínimo legal vigente (SMLV) que establece la ley laboral en el país. El valor de matrícula mas frecuente es del 1,5 SMLV con un porcentaje del $38.40 \%$, y el que menor solicitudes presenta es el del valor de un 3,5 SMLV con un índice de $1.44 \%$.

\begin{tabular}{|l|r|}
\hline \multicolumn{2}{|c|}{ SMLV que cancela como matrícula } \\
\hline 0.75 & 29 \\
\hline 1 & 17 \\
\hline 1,5 & 53 \\
\hline 1,75 & 4 \\
\hline 2 & 16 \\
\hline 2,5 & 7 \\
\hline 3 & 6 \\
\hline 3,5 & 2 \\
\hline$>3,5$ & 4 \\
\hline
\end{tabular}

Tabla 2. Relación de salarios mínimos legales vigentes que cancela como matrícula

Variables demográficas: estas variables indican características sociales y de identificación particular de los estudiantes, necesaria en la determinación o construcción de programas que garanticen el bienestar de las necesidades evidenciadas. 


\section{Ecomatemático}

Edad: la edad de los y las estudiantes que solicitan reliquidación está en el rango de 15 a 20 años de edad con un porcentaje de $61 \%$, y el de menor número de participación es el rango de edad mayor de 26 años en adelante.

\begin{tabular}{|c|c|}
\hline \multicolumn{2}{|c|}{ EDADES } \\
\hline $15-20$ años & 84 \\
\hline $21-25$ años & 41 \\
\hline 26 en adelante & 14 \\
\hline
\end{tabular}

Tabla 3. Relación de las edades

Género: del total de 138 estudiantes, el 51\% es de género masculino, mientras que un $49 \%$ es de género femenino.

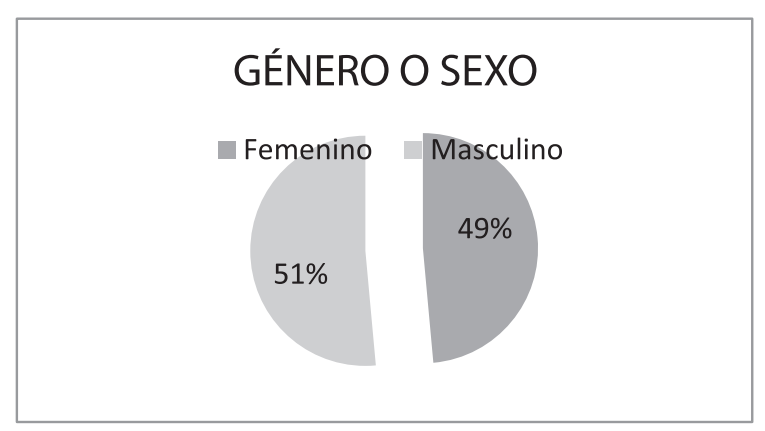

Gráfica 4. Distribución de género o sexo

Estado civil: el estado civil más frecuente de los estudiantes solicitantes es el de soltero(a) con un $83 \%$, mientras el que menor porcentaje presenta es el de casado(a) con un $1 \%$.

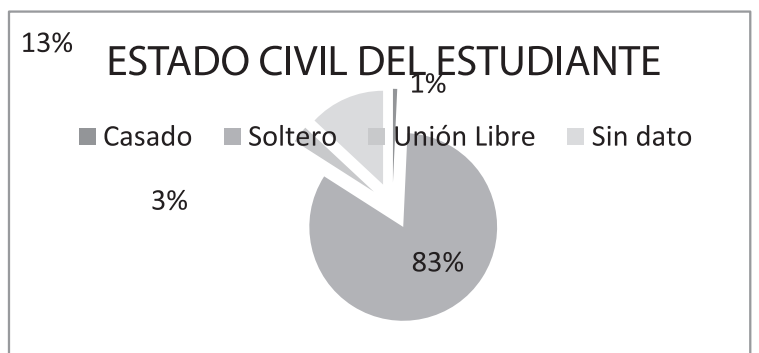

Gráfica 5. Distribución Estado civil del estudiante

Tipología familiar: el núcleo familiar del estudiante determina incluso las relaciones con el entorno social y económico, donde el mayor porcentaje se evidencia en la familia de tipología extensa con un
$29 \%$, y donde la tipología de menor incidencia es la recompuesta con un 3\% del total de estudiantes solicitantes. Sin embargo se identifica que un 10\% de los estudiantes vive lejos de su núcleo familiar o solo(a).

\begin{tabular}{|c|c|}
\hline \multicolumn{2}{|c|}{$\begin{array}{c}\text { TIPOLOGÍA FAMILIAR DEL } \\
\text { ESTUDIANTE }\end{array}$} \\
\hline Nuclear & Extensa \\
\hline Monoparental & Recompuesta \\
\hline \multicolumn{2}{|c|}{ El estudiante vive solo $\square$ Sin dato } \\
\hline $13 \% 10 \%$ & $23 \% 29 \%$ \\
\hline
\end{tabular}

Gráfica 6. Identificación de tipologías familiares

Relaciones familiares: de acuerdo a la tipología de la familia muchos de los componentes familiares son derivados, este especialmente lo constituyen la las relaciones y dinámicas familiares, su comunicación y la forma de disolver los conflictos. Según la información obtenida, la condición más frecuente es la unida con un $70 \%$, mientras la menos frecuente es la no existente o distante con un $2 \%$, sin embargo es particular identificar que con un porcentaje del 20\% declara tener relaciones conflictivas con su núcleo familiar.

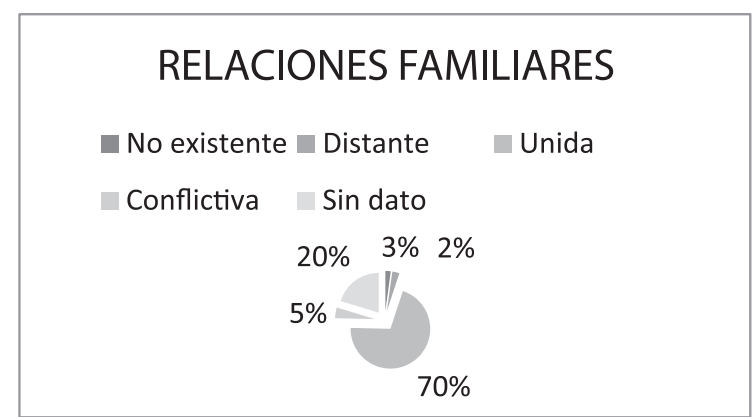

Gráfica 7. Relaciones familiares

Ocupación laboral del estudiante: la característica laboral del estudiante es también determinante del valor de matrícula, por lo que evidentemente el mayor número de estudiantes declara no trabajar con un porcentaje del $65 \%$, mientras solo un $13 \%$ afirman trabajar en jornada diurna comúnmente, en horarios alternos (fines de semana) y por temporadas. 


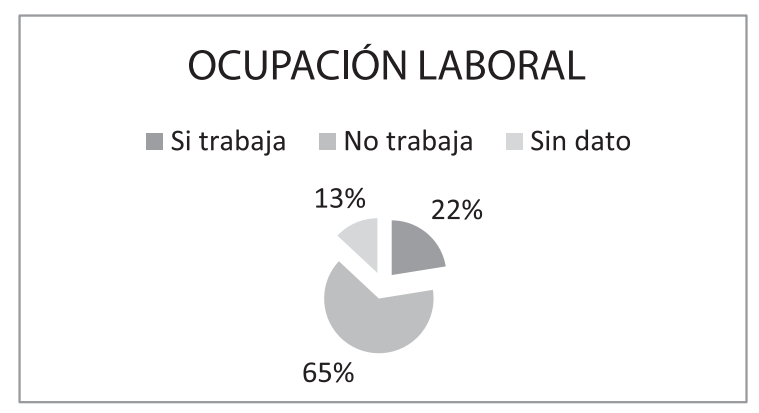

Gráfica 8. Ocupación laboral

Ingresos familiares: esta es una de las condiciones que estipula el valor de matrícula del estudiante, como lo indica el acuerdo 071 donde se indican los valores considerados para el proceso de reliquidación del valor de matrícula. Según la información obtenida el 50\% de las familias de los estudiantes afirma sostener económicamente su núcleo familiar con menos o hasta un SMLV, mientras solo un 9\% declara contar con más de tres SMLV para la manutención de su núcleo familiar.

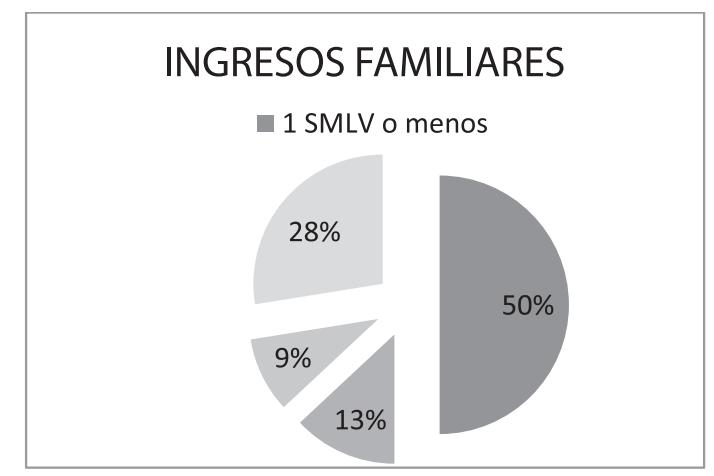

Gráfica 9. Ingresos familiares

Variables geográficas: las variables geográficas determinan la condición social y económica principalmente con la que el estudiante convive, condiciones que son de suma importancia para identificación espacial y del entorno donde los estudiantes y sus familias se relacionan, desarrollan y conviven con su entorno.

Lugar de residencia: en relación al lugar de residencia, se tuvo en cuenta la delimitación por comunas que el área metropolitana de Cúcuta posee, así como la inclusión de otros municipios residencia de los estudiantes. La delimitación del área metropolitana que se empleó fue:
Comuna 1 (Centro): comuna del Centro de Cúcuta, donde predomina la zona comercial, en particular centros comerciales como Alejandría, San Andresito, Ventura Plaza, entre otros. Aunque se encuentran algunos barrios residenciales, entre ellos Los Caobos y La Playa. Esta zona está conformada por barrios como: El Contento, El Páramo, El Centro, La Playa, Latino, El Callejón, Sector la Sexta, entre otros.

Comuna 2 (Centro-Oriental): zona conformada por barrios como: La Rinconada, El lago, Club Tenis, Comercial Bolívar, El Rosal, Barrio Blanco, Los Caobos, Quinta Vélez, La Rivera, El Colsag, Popular, La Ceiba, Santa Lucia, Los Acacios, Prados I y II, Prados Club, Brisas del Pamplonita, Rincón del prado, Valparaíso suite, Las Almeidas, La primavera, Manolo Lemus, Condado de castilla, CASD, y el sector aledaño a la Universidad Francisco de Paula Santander.

Comuna 3 (Sur-Oriental): conformada por los barrios denominados: Boconó, Santa Ana, La Unión, Valle Esther, Policarpo, Las Margaritas, Aguas Calientes, La Libertad, San Mateo, Bogotá, Bellavista, y La Carolina.

Comuna 4 (Oriental): conformada por los barrios denominados: Nuevo Escobal, La Quinta, El Escobal, El Portal del Escobal, Isla de la Fantasía, La Alameda, El Higuerón, San Martín, Urbanización San José, Urbanización Aniversario, y Nueva Santa Clara.

Comuna 5 (Nor-Oriental): conformada por los barrios denominados: Lleras, La Merced, Colpet, Pescadero, Zona Industrial, Urbanización El Bosque, Prados del Norte, Tasajero, Niza, Zona Franca, Santa Helena, Zulima I, II, III y IV Etapa, Gratamira, La Mar, Ciudad Jardín, Guaimaral, Ceiba II, San Eduardo I y II, y Gualanday.

Comuna 6 (Norte): conformada por los barrios denominados: Virgilio Barco, Porvenir, Alonsito, San Gerardo, Aeropuerto, Panamericano, El Salado, La Ínsula, Colinas de la Victoria, Sevilla, Cerro Norte, Cerro de la Cruz, Las Américas, y Camilo Daza.

Comuna 7 (Nor-Occidental): conformada por los barrios denominados: El Rosal del Norte, Chapinero, Comuneros, El Claret, Tucunaré, Motilones, La Florida, La Primavera, Ospina Peréz, Bue- 


\section{Ecomatemático}

nos Aires, La Hermita, Paraíso, y Camilo.

Comuna 8 (Occidental): conformada por los barrios denominados: El Progreso, Antonia Santos, Cerro Pico, Palmeras, Belisario, Atalaya, I, II y III etapa, Cúcuta 75, Los Almendros, Carlos Ramírez París, Doña Nidia, La Victoria, Kennedy, y El Rodeo.

Comuna 9 (Sur-Occidental): conformada por los barrios denominados: Divina Pastora, Belén, Rudensindo Soto, Gaitán, Barrio Nuevo, La Aurora, Las Colinas, Cundinamarca, San Miguel, Loma de Bolívar, Los Alpes, Carora, y Pueblo Nuevo.

Comuna 10 (Cementerio): conformada por los barrios denominados: Cementerio, San José, Circunvalación, Camilo Torres, La Cabrera, Puente Barco, Santo Domingo, San Rafael, Alonso López, Galán, Santander, Las Malvinas, El Cortijo, Grupo Maza y Coca-cola.

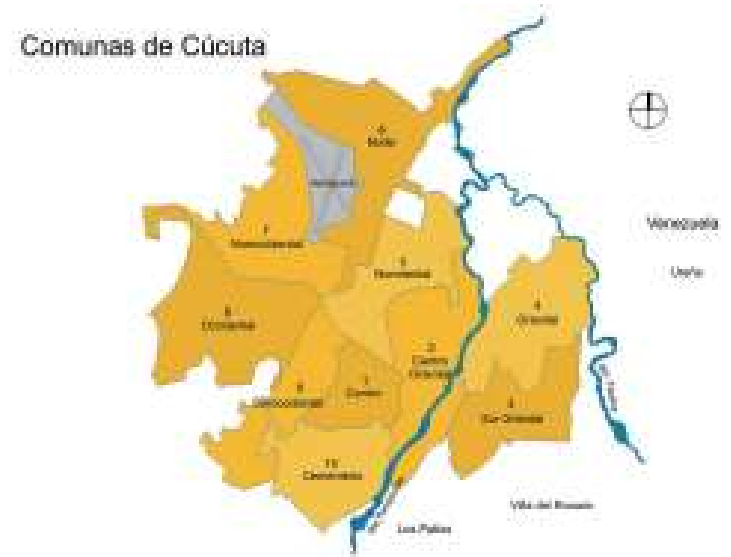

Gráfica 10. Mapa del municipio de Cúcuta por comunas ${ }^{1}$

De acuerdo a esta delimitación espacial, los barrios de mayor afluencia de residentes de la Universidad Francisco de Paula Santander, son la comuna 3 y la comuna 5 , residencias de estrato socio-económico 3 a 5, dando un margen estándar en calidad de vida para las condiciones de la vivienda. Uno de los sectores de menor afluencia de residentes es el perteneciente a la comuna 10 , sector cementerio de la ciudad, donde además se resalta la incidencia de otros municipios en la residencia de los estudiantes y su núcleo familiar, en municipios como Villa del Rosario y Los Patios, incluso municipios del país vecino, Venezuela.

\begin{tabular}{|lr|}
\hline \multicolumn{2}{|c|}{ LUGAR DE RESIDENCIA } \\
\hline Comuna 1 & 4 \\
\hline Comuna 2 & 9 \\
\hline Comuna 3 & 27 \\
\hline Comuna 4 & 3 \\
\hline Comuna 5 & 28 \\
\hline Comuna 6 & 6 \\
\hline Comuna 7 & 6 \\
\hline Comuna 8 & 20 \\
\hline Comuna 9 & 12 \\
\hline Comuna 10 & 0 \\
\hline $\begin{array}{l}\text { Otro municipio de N. } \\
\text { de S. }\end{array}$ & 20 \\
\hline Venezuela & 1 \\
\hline Sin dato & 2 \\
\hline
\end{tabular}

Tabla 4. Relación de lugar de residencia

Estrato socioeconómico: la condición socioeconómica de los barrios y comunas donde residen los estudiantes, identifica las condiciones habitacionales de las viviendas, donde el estrato más común entre los estudiantes solicitantes es el 2 (bajo) que indica comúnmente factores de escases económica, sin embargo su habitabilidad es dependiente de las condiciones de la vivienda, debido a que el sector puede ser una zona de tránsito. Además, evidentemente los estratos favorecidos económicamente (4 en adelante), son los de menor coincidencia.

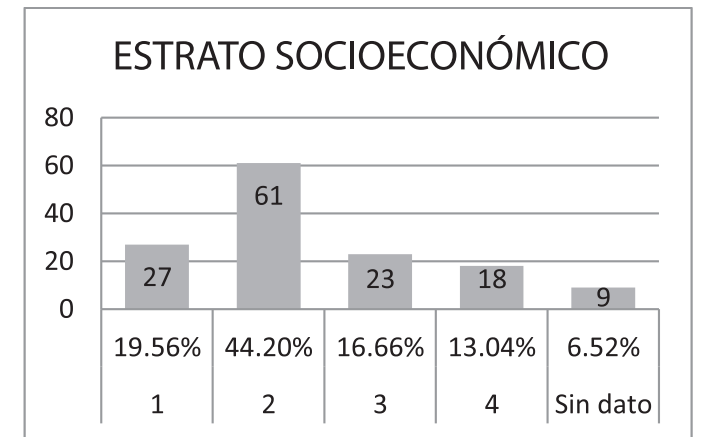

Gráfica 11. Estrato socioeconómico 


\section{Ecomatemático}

Lugar de nacimiento: El lugar de nacimiento del estudiante, es primordial a la hora de definir el pasado familiar y la movilidad de su núcleo. Luego del municipio de Cúcuta con un $65,93 \%$, se rescatan valores como los pertenecientes a otros municipios del departamento de Norte de Santander (14.49\%), así como de otros departamentos del país (15\%), e incluso del exterior (3.62\%).

\begin{tabular}{|lcc|}
\hline & \multicolumn{1}{c|}{$\begin{array}{c}\text { LUGAR DE } \\
\text { NACIMIENTO }\end{array}$} \\
\hline $\begin{array}{l}\text { Municipio de } \\
\text { Cúcuta }\end{array}$ & 91 & $65,93 \%$ \\
\hline $\begin{array}{l}\text { Otro municipio de } \\
\text { N. de S. }\end{array}$ & 20 & $14,49 \%$ \\
\hline $\begin{array}{l}\text { Departamento del } \\
\text { Cesar }\end{array}$ & 9 & $6,52 \%$ \\
\hline $\begin{array}{l}\text { Departamento de } \\
\text { Arauca }\end{array}$ & 4 & $2,89 \%$ \\
\hline $\begin{array}{l}\text { Otro Departamento } \\
\text { del País }\end{array}$ & 9 & $6,52 \%$ \\
\hline Exterior & 5 & $3,62 \%$ \\
\hline
\end{tabular}

Tabla 5. Relación de lugar de nacimiento

Tipo de vivienda: la característica de la vivienda donde residen los estudiantes, así como su dotación, describe la condición en la que habita el núcleo familiar, donde la mayor parte $(58.69 \%)$ reside en casas, de una planta, con al menos una habitación y un baño, donde además tienen condicionado espacios de preparación y consumo de alimentos, así como de esparcimiento y recibo de visitas. Sin embargo, por la afluencia de estudiantes foráneos a la institución, la mayor parte de estos residen en habitaciones con los mismos servicios de un domicilio familiar, solo que con la característica de compartir estos espacios con hasta diez (10) personas más que no tienen ninguna relación familiar con el estudiante, incluso el núcleo familiar de cada uno de ellos se encuentra en la ciudad de origen del estudiante, con comunicación directa solo en épocas de vacaciones o recesos académicos.

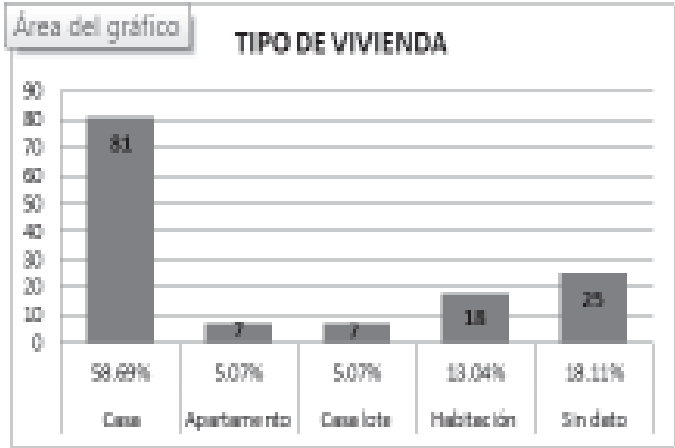

Gráfica 12. Tipo de vivienda

Tenencia de la vivienda: La condición más encontrada dentro del grupo de estudiantes solicitantes es la de vivienda propia con un $42.02 \%$, siendo la segunda con más incidencia la condición de arriendo con un $30.43 \%$, y siendo la de menor número de coincidencias el subarriendo y la invasión con un $0.72 \%$ cada uno de estos.

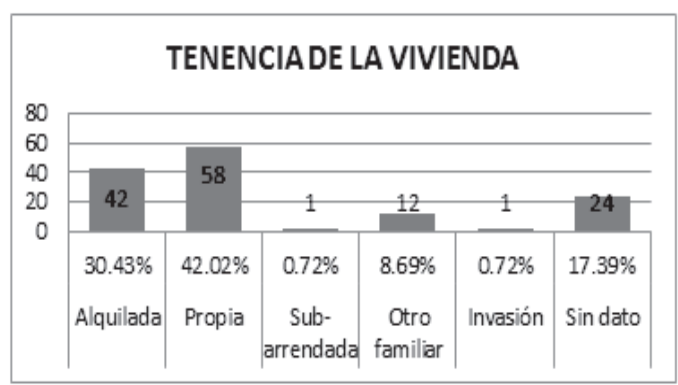

Gráfica 13. Tenencia de la vivienda

Servicios públicos: Los servicios públicos más comunes de acceso en los estudiantes son acueducto y alumbrado público (83.33\%), y los de menor acceso y por ende que requieren de mayor valor adquisitivo son las telecomunicaciones (telefonía fija, televisión por cable e internet) con un 38.40\% y el gas domiciliario con un $45.65 \%$.

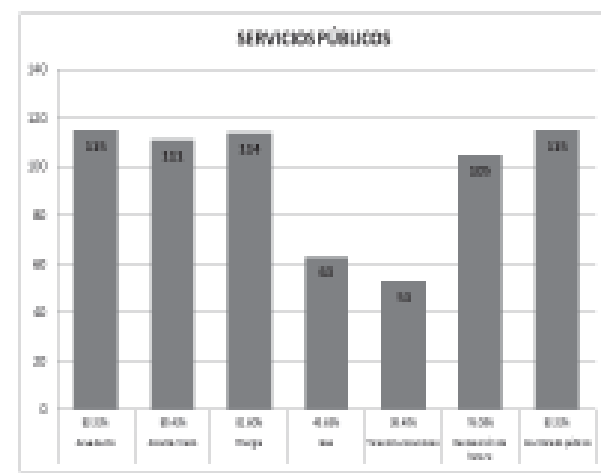

Gráfica 14. Servicios públicos 


\section{Ecomatemático}

Materiales de la vivienda: los materiales de la vivienda, están relacionados con el sector donde se encuentra ubicada la vivienda, su poder adquisitivo y gustos o concepción de habitabilidad y comodidad. En paredes el material más encontrado es el ladrillo de arcilla o bloque con un $77.53 \%$, y el menos encontrado es el denominado otros materiales además de ladrillo, madera y bareque, con $1.44 \%$.

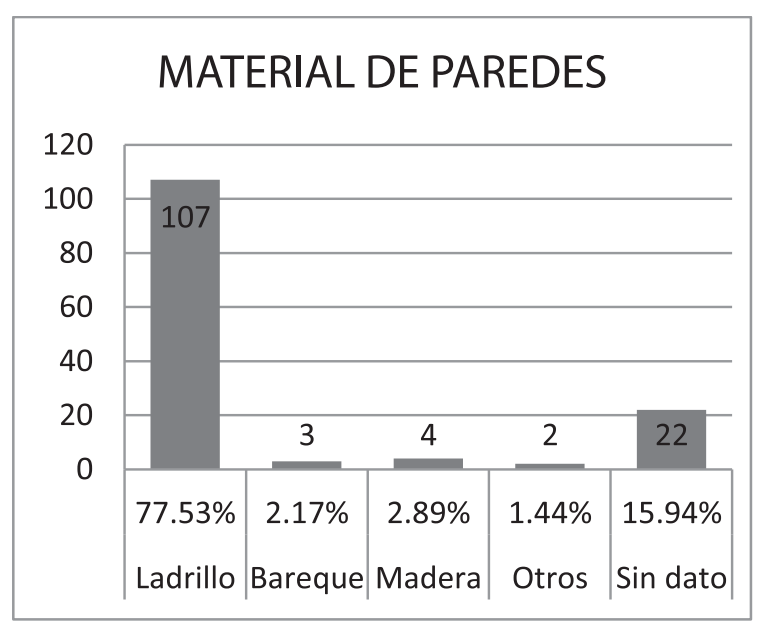

Gráfica 15. Material de paredes

El material de los pisos más común dentro de los estudiantes solicitantes es la baldosa o tableta con un $65.21 \%$, y el de menor número de incidencia es tierra con un $4.34 \%$.

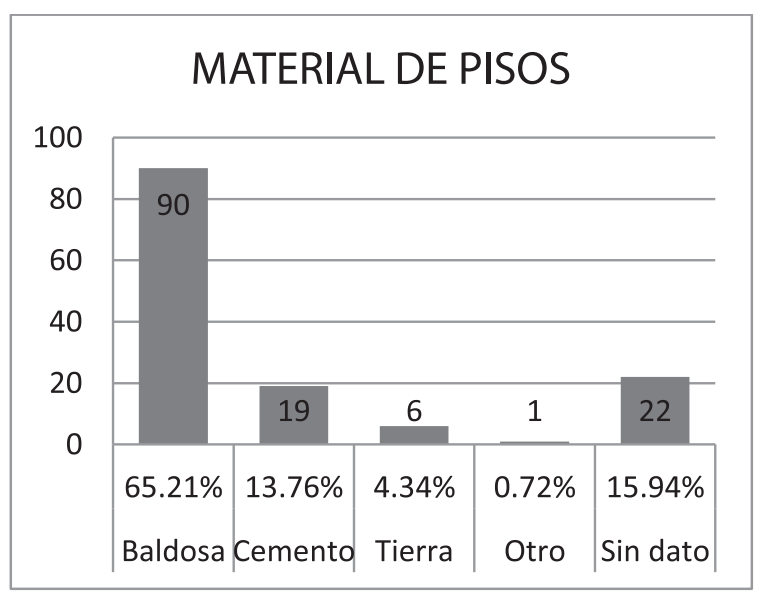

Gráfica 16. Material de pisos

El material de los techos encontrados en las viviendas visitadas se caracterizó por que, el de mayor coincidencia fue la placa con un $29.71 \%$, sobre el eternit, segundo en incidencia con un $28.98 \%$, siendo el de menor número de coincidencia otro tipo de materiales para el techado de las viviendas.

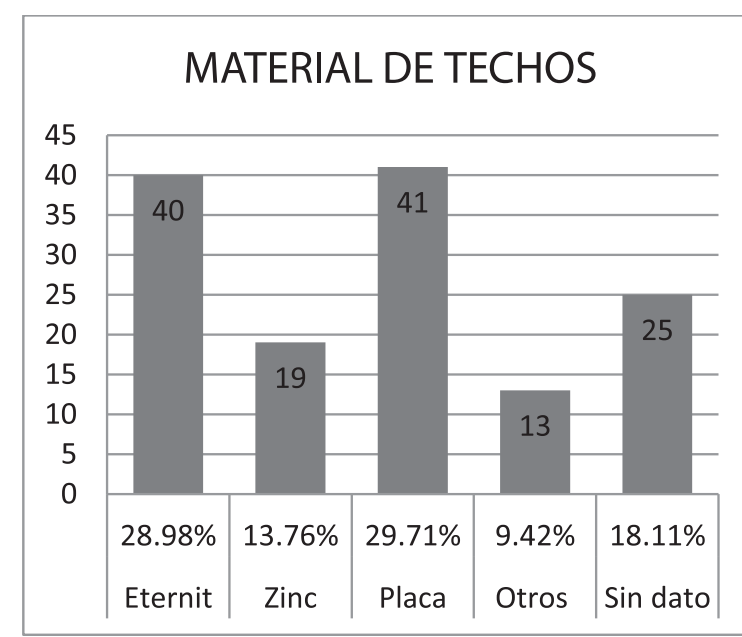

Gráfica 17. Material de techos

\section{Conclusiones}

La matriz DOFA, muestra conclusiones y la relación del espacio educativo de la UFPS y las condiciones socioeconómicas de los estudiantes objeto de estudio. 
Ecomatemático

\begin{tabular}{|c|c|c|c|c|}
\hline ACTOR & FORTALEZAS & OPORTUNIDADES & DEBILIDADES & AMENAZAS \\
\hline $\begin{array}{c}\text { Universidad } \\
\text { Francisco de } \\
\text { Paula Santander, } \\
\text { y su dependencia } \\
\text { dispuesta para } \\
\text { estos fines La } \\
\text { Vicerrectoría de } \\
\text { Bienestar } \\
\text { Universitario }\end{array}$ & 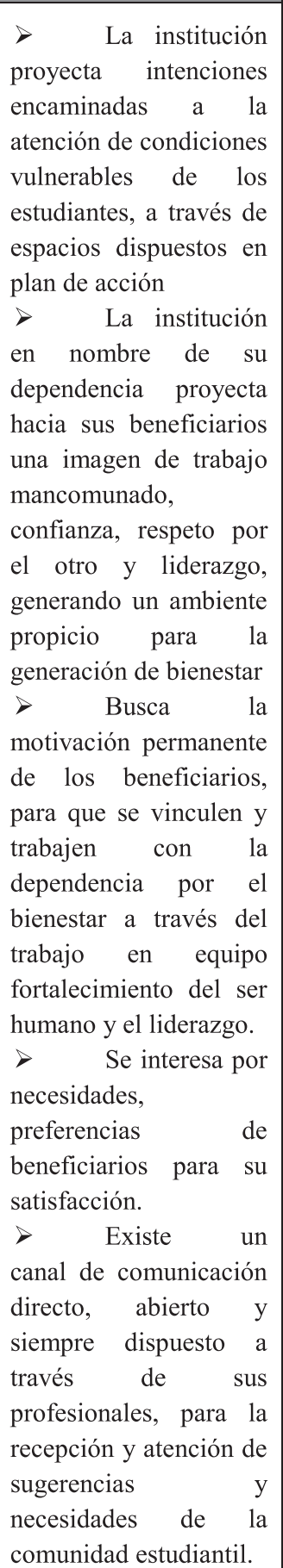 & 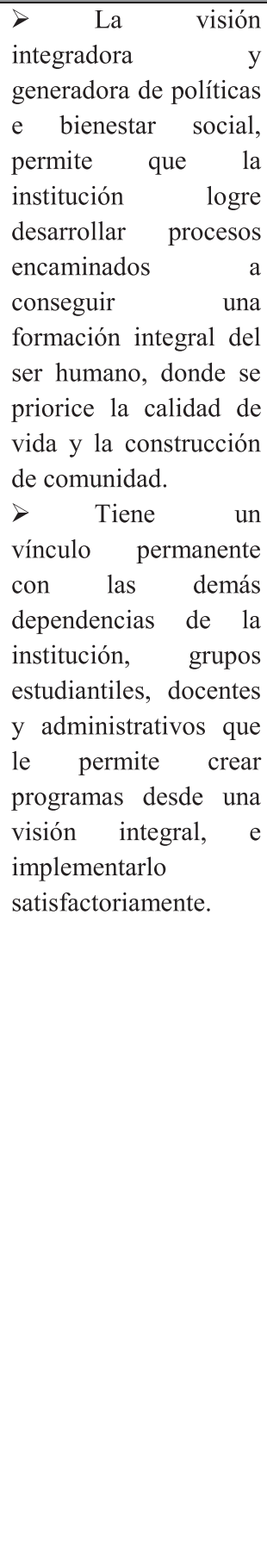 & $\begin{array}{lr} & \text { La magnitud } \\
\text { de la población a } & \\
\text { atender y los } & \text { o } \\
\text { colaboradores } & \text { orofesionales } \\
\text { proscritos a ella, } \\
\text { resultan } \\
\text { insuficientes para la } \\
\text { atención total de sus } \\
\text { beneficiarios, lo cual } \\
\text { puede llevar a } \\
\text { condicionar el trabajo } \\
\text { e intervención de los } \\
\text { colaboradores, al } \\
\text { contar con tiempos } \\
\text { parcializados, poco } \\
\text { impacto poblacional } \\
\text { atendido, y hasta } \\
\text { desgaste del recurso } \\
\text { humano y de procesos } \\
\text { que se llevan a cabo. }\end{array}$ & $\begin{array}{l}\text { La institución } \\
\text { y esta dependencia } \\
\text { como responsable de } \\
\text { dichos procesos, y con } \\
\text { condiciones poco } \\
\text { favorables de impacto } \\
\text { en la población objeto, } \\
\text { incide en los } \\
\text { indicadores } \\
\text { desarrollo del } \\
\text { efectividad } \\
\text { proyectos y estrategias } \\
\text { que establecidas para } \\
\text { atender y cumplir con } \\
\text { la visión que se } \\
\text { estableció como su fin. }\end{array}$ \\
\hline $\begin{array}{l}\text { ACTOR SABER } \\
\text { (División de } \\
\text { Servicios } \\
\text { Asistenciales y } \\
\text { de Salud) }\end{array}$ & 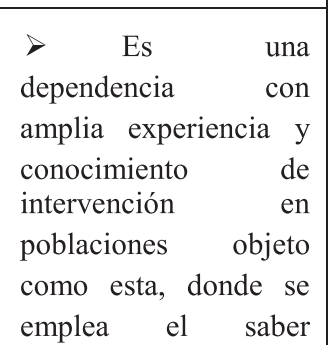 & $\begin{array}{l}>\text { La permanente } \\
\text { programación de } \\
\text { procesos, proyectos, } \\
\text { campañas y estudios } \\
\text { como este sobre y para } \\
\text { sus beneficiarios, } \\
\text { permite } \\
\text { conocimiento a fondo }\end{array}$ & $\begin{array}{l}\text { Es evidente } \\
\text { la falta y necesidad de } \\
\text { profesionales } \\
\text { capacitados, con } \\
\text { disposición de tiempo } \\
\text { y con interés en el } \\
\text { trabajo con } \\
\text { comunidades como la }\end{array}$ & \begin{tabular}{lrr} 
La & \multicolumn{2}{r}{ realidad } \\
cambiante de las \\
condiciones & sociales, \\
económicas & & $y$ \\
& & \\
culturales de los & lon \\
beneficiarios, es de & \\
constante trabajo y \\
monitoreo, de jornadas
\end{tabular} \\
\hline
\end{tabular}




\begin{tabular}{|c|c|c|c|c|}
\hline & 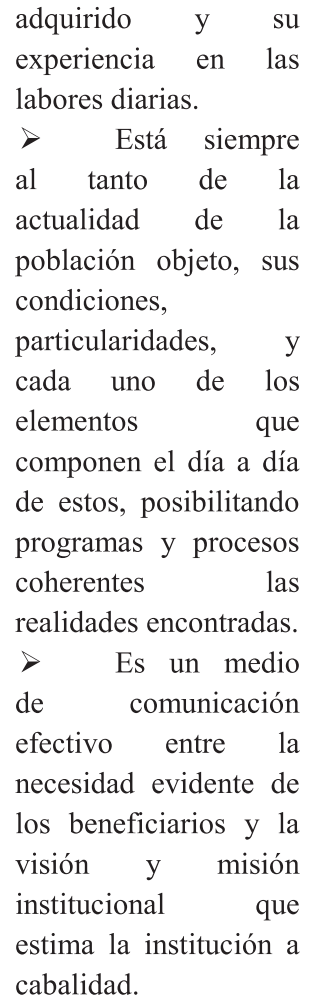 & $\begin{array}{l}\text { de la realidad social y } \\
\text { económica de los } \\
\text { estudiantes. }\end{array}$ & $\begin{array}{l}\text { universitaria, ya que } \\
\text { debido a esto han } \\
\text { atrasado, dado por } \\
\text { inconcluso e incluso } \\
\text { por poco efectivo los } \\
\text { procesos que han } \\
\text { adelantado con tanto } \\
\text { esfuerzo de su poco } \\
\text { personal. }\end{array}$ & 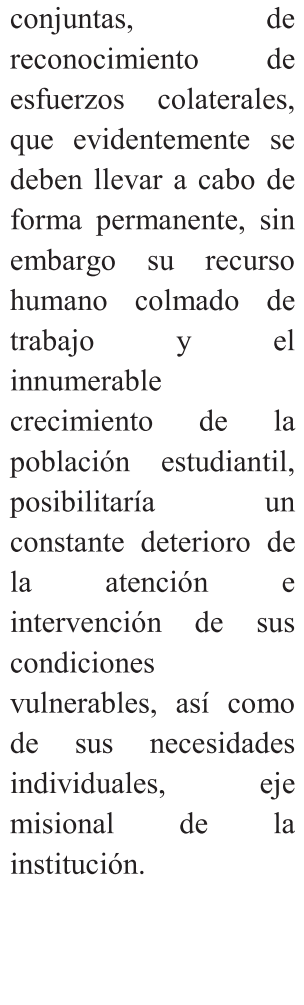 \\
\hline $\begin{array}{c}\text { ACTOR } \\
\text { SOCIAL Y } \\
\text { BENEFICIARIO } \\
\text { (Estudiantes } \\
\text { solicitantes de } \\
\text { reliquidación del } \\
\text { valor de su } \\
\text { matrícula) }\end{array}$ & 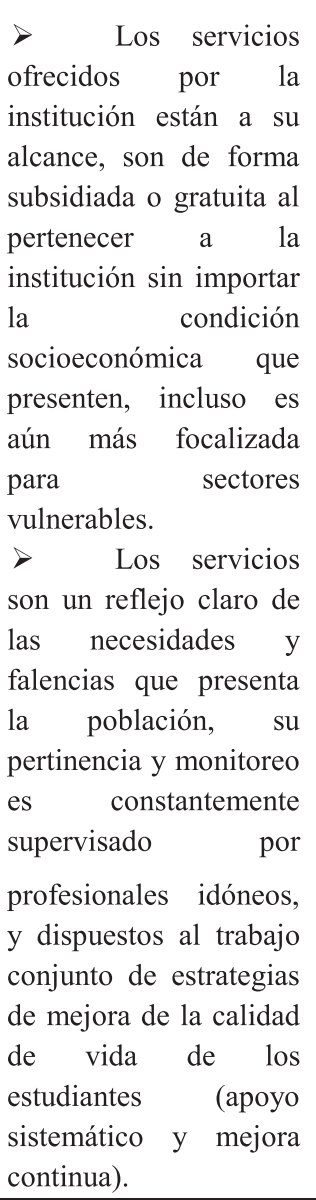 & 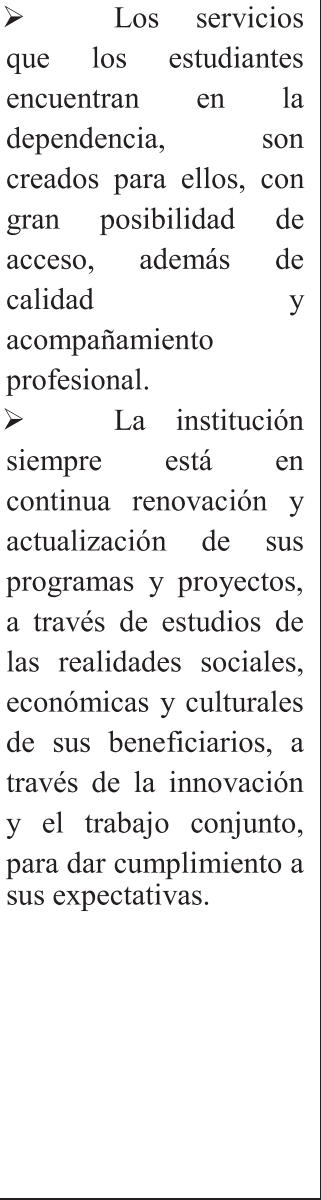 & 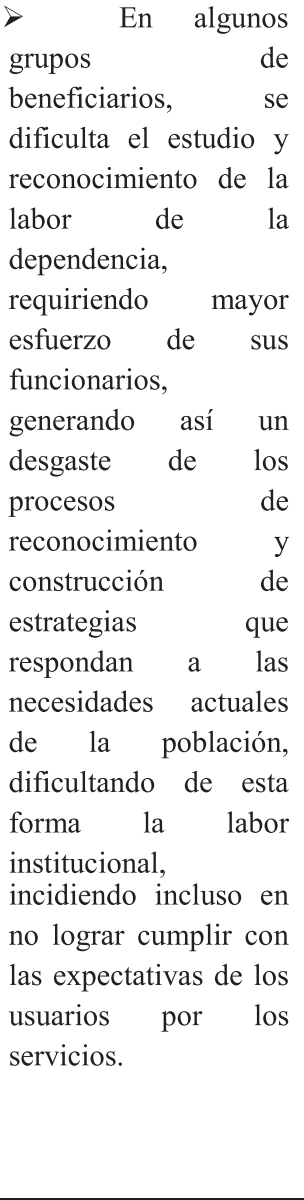 & $\begin{array}{l}\text { El desinterés } \\
\text { por participar y falta } \\
\text { de información de los } \\
\text { servicios que ofrece la } \\
\text { dependencia } \\
\text { específicamente para } \\
\text { los estudiantes, genera } \\
\text { un poco afluencia de } \\
\text { participantes de los } \\
\text { servicios ofrecidos, } \\
\text { incidiendo } \\
\text { permanentemente en } \\
\text { los indicadores de } \\
\text { impacto establecidos } \\
\text { para los servicios. }\end{array}$ \\
\hline
\end{tabular}




\section{Ecomatemático}

- A pesar de las constantes solicitudes de reliquidación que se presentan, donde el valor de matrícula es bajo, es importante considerar la remisión de estas solicitudes a otras dependencias que evalúen las condiciones del estudiante y su núcleo familiar, así como facilitar otras posibilidades de reducción del valor de la matrícula académica, para que de esta forma sea un servicio de atención integral y no asistencial.

- La edad, así como el semestre que cursan, pueden ser indicadores de la falta de información de los servicios y procesos que adelanta la dependencia en busca del bienestar integral y la mejora de la calidad de vida de los estudiantes, aspecto importante para la incidencia e importancia de reconocimiento del trabajo de la dependencia.

- La tipología familiar, las relaciones que se desarrollan en sus núcleos, la ocupación laboral, el lugar de nacimiento y residencia, son insumos fundamentales para la implementación de nuevos procesos o para el mejoramiento o direccionamiento de los ya existentes, que al ser vulnerables a cambios en condiciones de vulnerabilidad, deben ser considerados y actualizados permanentemente.

- Las relaciones familiares y la tipología familiar, son indicadores de necesidades primarias de los estudiantes, que al ser este un espacio primordial para el desarrollo integral de estos, requiere de mayor acompañamiento individual y social del sujeto y su familia, orientación psicológica, capacitaciones y talleres de crecimiento individual y colectivo (grupo familiar), así como el fortalecimiento de las relaciones sociales.

- El deseo de superación ha llevado a algunos estudiantes universitarios a buscar empleos informales, sin embargo, los ingresos bajos no les permite cubrir costos de matrícula.

- La educación considera a los estudiantes universitarios que solicitan la reliquidación de matrícula es el medio adecuado para mejorar su situación económica y social. Sin embargo, es también la herramienta que permitiría perpetuar y acentuar las desigualdades. El resultado dependerá de los recursos que se des- tinen a la educación, y de las políticas sociales específicas que reduzcan los costos de oportunidad para estudiantes de clases bajas.

\section{Bibliografía y cibergrafía}

Bolívar, Antonio. 2006. Familia y escuela, dos mundos llamados a trabajar en común. Revista de Educación 339: 119-14

Campos Ríos, Guillermo. 2002. Implicancias del concepto de empleabilidad en la reforma educativa. Revista Iberoamericana de Educación, 1-9.

Constitución de la República de Colombia. Editorial Mejoras. Barranquilla, Colombia. 2000.

CEPAL. 2009. Estudio económico deAmérica Latina y el Caribe 2008-2009. < http://www.eclac.org/ publicaciones/xml/4/36464/2009-483-EEE_2009completo-WEB.pdf $>$.

CIFUENTES, Rosa María. "Conceptos para leer la intervención de Trabajo Social, aporte a la construcción de realidad".

IBARRA RUSSI, Oscar Armando. "Estudio de caracterización: estudiantes admitidos primer semestre 2006". Universidad Pedagógica Nacional. Bogotá. Colombia. 2007.

JIMÉNEZ BENÍTEZ, William. "El enfoque de derechos humanos y las políticas públicas". Universidad Sergio Arboleda. Bogotá. Colombia. 2007.

Morduchowicz, Alejandro. 2003. Discusiones de economía de la educación. Buenos Aires: Editorial Losada.

Rus, Tomás. 2012. Efectos de la duración del desempleo en las actitudes hacia el trabajo de los mayores de 45 años. Revista Electrónica de Investigación y Docencia (REID) 8: 7-21.

Universidad Francisco de Paula Santander. Vicerrectoría de Bienestar Universitario. División de Servicios Asistenciales y de Salud. "Caracterización Socioeconómica de la población estudiantil que ingresa a la UFPS en el primer semestre del año 2005 jornada diurna modalidad presencial. Cúcuta. Colombia. 2005. 
Weinberg, Pedro. 2004. Formación profesional, empleo y empleabilidad. < http://www.cinterfor. org.uy/ public/spanish/region/ampro/cinterfor/ $\mathrm{publ} / \mathrm{sala} /$ weinberg/index.htm $>$.

\section{Cibergrafía:}

Información institucional. Misión y Visión. Encontrado en: www.ufps.edu.co. Consultado el: 20 de Enero de 2013.

Estatuto general: acuerdo superior № 1 del de Marzo de 1994. Tomado de: http:// www.udea.edu.co/portal/page/portal/portal/a. InformacionInstitucional/a.QuienesSomos/A.naturalezaJuridicaDomicilio. El 20 de Enero de 2013.

Información institucional. Historia: UFPS. Encontrado en: www.ufps.edu.co. Consultado el: 20 de Enero de 2013.

Vicerrectoría de Bienestar Universitario. Información institucional. Encontrado en: www.ufps.edu. co. Consultado el: 20 de Enero de 2013.

Ministerio de las Tecnologías de la información y las comunicaciones. República de Colombia. Gobierno en línea. Guía para la caracterización de usuarios de las entidades públicas. Tomado de: www.mintic.gov.co.Consultado el: 15 de Marzo de 2013. 